\title{
Fullerene Coalescence in Nanopeapods: A Path to Novel Tubular Carbon
}

\author{
E. Hernández, ${ }^{\dagger}$ V. Meunier, ${ }^{\ddagger}$ B. W. Smith,, R. Rurali,,$"$ H. Terrones,${ }^{\perp}$ \\ M. Buongiorno Nardelli, ${ }^{\ddagger \neq},{ }^{\prime}$ M. Terrones, ${ }^{,, \perp}$ D. E. Luzzi, ${ }^{\S}$ and J.-C. Charlier ${ }^{\nabla}$
}

Institut de Ciència de Materials de Barcelona (ICMAB-CSIC), Campus de Bellaterra, 08193 Barcelona, Spain, Computer Science and Mathematics Division, Oak Ridge

National Laboratory, Oak Ridge, Tennessee 37831, Department of Materials Science

and Engineering, University of Pennsylvania, Philadelphia, Pennsylvania 19104,

Centre Nacional de Microelectrònica (CNM-CSIC), Campus de Bellaterra,

08193 Barcelona, Spain, Advanced Materials Department, IPICYT, Av. Venustiano

Carranza 2425-A, San Luis Potosí 78210, México, Department of Physics,

North Carolina State University, Raleigh, North Carolina 27695, and Université

Catholique de Louvain, PCPM \& CERMIN, B-1348 Louvain-la-Neuve, Belgium

Received May 5, 2003; Revised Manuscript Received May 12, 2003

\section{ABSTRACT}

A fascinating structural transformation occurring inside single-walled carbon nanotubes (SWNTs) is the fullerene coalescence, which is responsible for forming stable zeppelinlike carbon molecules. We report in situ transmission electron microscope (TEM) observations revealing sequences of fullerene coalescence induced by electron irradiation on pristine nanotube peapods, together with extensive theoretical investigations of the microscopic mechanism underlying this process. TEM images indicate that the merging of fullerenes results in stable but corrugated tubules (5 to $7 \AA$ in diameter) confined within SWNTs. These observations have been confirmed using a combination of theoretical approaches based on molecular dynamics, empirical potentials, tight-binding methods, Monte Carlo techniques, and first principles calculations. We have fully elucidated the coalescence mechanism of fullerenes inside SWNTs under electron irradiation and thermal annealing. The process occurs via the polymerization of $\mathrm{C}_{60}$ molecules followed by surface reconstruction, which can be triggered either by the formation of vacancies (created under electron irradiation) or by surface-energy minimization activated by thermal annealing. These novel tubular forms of carbon contain hexagons, pentagons, heptagons, and octagons. The stability, electronic properties, and electron conductance of the novel tubules are strongly affected by the final geometry of the coalesced fullerene complex. The possibility of forming highly conducting and semiconducting tubular structures suggests new avenues in designing carbon nanowires with specific electronic characteristics.

Research on carbon nanostructures developed rapidly after the discovery of $\mathrm{C}_{60}$ in the mid $1980 \mathrm{~s}^{1}$ and the identification of carbon nanotubes in the early 1990s. ${ }^{2}$ The structural diversity of carbon has been confirmed even further by the discovery of nanopeapods (NPPs), ${ }^{3-7}$ a hybrid structure consisting of fullerene molecules encapsulated in SWNTs. Along the same lines, the production and characterization of encapsulated endohedral fullerenes (e.g., $\mathrm{Gd} @ \mathrm{C}_{82},{ }^{8-10}$ $\mathrm{La}_{2} @ \mathrm{C}_{80}{ }^{11}$ ) within SWNTs has opened a broad avenue in the fabrication of quantum memory and computing devices. Recently, the induced coalescence of $\mathrm{C}_{60}$ molecules in hot vapors using mass spectrometry ${ }^{12}$ and fullerenes encapsulated

\footnotetext{
* Corresponding author. E-mail: mterrones@ipicyt.edu.mx.

Institut de Ciència de Materials de Barcelona.

$\doteqdot$ Oak Ridge National Laboratory.

$\S$ University of Pennsylvania.

"Centre Nacional de Microelectrònica.

$\perp$ IPICYT.

\# North Carolina State University.

$\nabla$ Université Catholique de Louvain.
}

in nanotubes (by electron irradiation or thermal annealing $)^{3-5}$ has revealed the possibility of creating nanotubes of extremely small diameter (e.g., 4-7 ̊). These small-diameter tubules, embedded in zeolite matrices, have been found to exhibit superconductivity at ca. $15-20 \mathrm{~K},{ }^{13}$ thus demonstrating that the coalescence of fullerene molecules inside SWNTs or other confining environments may provide a unique opportunity to design a new generation of tubular nanodevices exhibiting as-yet unknown properties. ${ }^{14}$

Recent theoretical studies on the formation of NPPs have shown that in order to enter the nanotube core the $\mathrm{C}_{60}$ molecules should possess specific energies. ${ }^{15}$ These energies were found to depend on the temperature at which NPPs form under thermal annealing (ca. 300-450 ${ }^{\circ} \mathrm{C}$ ). ${ }^{15}$ Other theoretical studies have described the merging of nanotubes by the caps using topological arguments. ${ }^{16}$ Tight-binding molecular dynamics simulations have shown that lateral nanotube coalescence is also possible if vacancies are created under high electron irradiation under annealing conditions. ${ }^{17}$ 
However, the coalescence mechanism of fullerenes in NPPs as well as the electronic and mechanical properties of the resulting tubules have not been reported hitherto.

In this article, we present the first theoretical study of $\mathrm{C}_{60}$ coalescence inside SWNTs induced by electron irradiation and/or thermal annealing. We have used a combination of techniques such as Monte Carlo (MC) and molecular dynamics (MD) in conjunction with empirical potentials, tightbinding methods, and ab initio electronic structure calculations to achieve a truly multiscale picture of various systems and prolonged simulation times. Our investigation reveals the spontaneous coalescence of the molecules into morestable tubular structures containing pentagonal, hexagonal, heptagonal, and octagonal carbon rings. Our molecular models agree with our experimental high-resolution transmission electron microscopy (HRTEM) observations. Finally, we discuss theoretical predictions of the electronic properties and stability, using tight-binding and ab initio approaches, of the tubular structures. The experimental measurements were performed using SWNTs synthesized by pulsed-laser vaporization and provided by NASA. Fullerene peapods were created from this starting material by the vapor-phase method, during which the SWNTs were vacuum annealed together with a $\mathrm{C}_{60}$ powder for $6 \mathrm{~h}$ at $650{ }^{\circ} \mathrm{C}$. Details of the synthesis of these structures have been discussed previously. ${ }^{4-5}$ The end result of this process is a free-standing "paper" of entangled SWNT peapods that is electron transparent at its periphery.

TEM samples were prepared without the use of solvents or a support film, which could have conceivably affected the response of the material to electron irradiation. Instead, a section of the peapod paper was fixed between the halves of a folding, low-mesh copper grid. Observation was performed at room temperature using a JEOL 2010F fieldemission microscope operating at $97 \mathrm{kV}$. Electron flux through the sample was approximately constant at $2.4(10)^{19}$ e $\mathrm{cm}^{-2} \mathrm{~s}^{-1}$, as calibrated by the current density registered on the imaging screens. Because the electron beam has a Gaussian intensity profile, this nominal value is the average over an area swept out by roughly $1 / 3$ of the total beam radius, and the actual dose rate to the sample may be higher or lower at specific points. It is noteworthy that the dose rate is nearly identical to that imposed in a prior irradiation study of empty SWNTs. ${ }^{18}$

Figure 1 shows the sequence of an isolated peapod undergoing continuous, constant irradiation while surrounded by vacuum. The time elapsed between each sequential image is $\sim 60-90 \mathrm{~s}$, corresponding to a total dose of approximately $10^{22} \mathrm{e} \mathrm{cm}^{-2}$ from $\mathrm{A}$ to $\mathrm{H}$. Initially visible in $\mathrm{B}$ is the formation of peanutlike fullerene "dimers," followed by a more advanced fusion of discrete molecules. The ultimate product is a series of corrugated, 5-7 $\AA$ diameter tubules that exhibit sites of negative curvature.

It has been previously demonstrated that $97 \mathrm{kV}$ is within the regime of ballistic damage for SWNTs, ${ }^{18}$ where the primary damage mode is the knock-on displacement of carbon atoms. Carbon atoms belonging to the interior fullerenes will be displaced at a higher rate than those

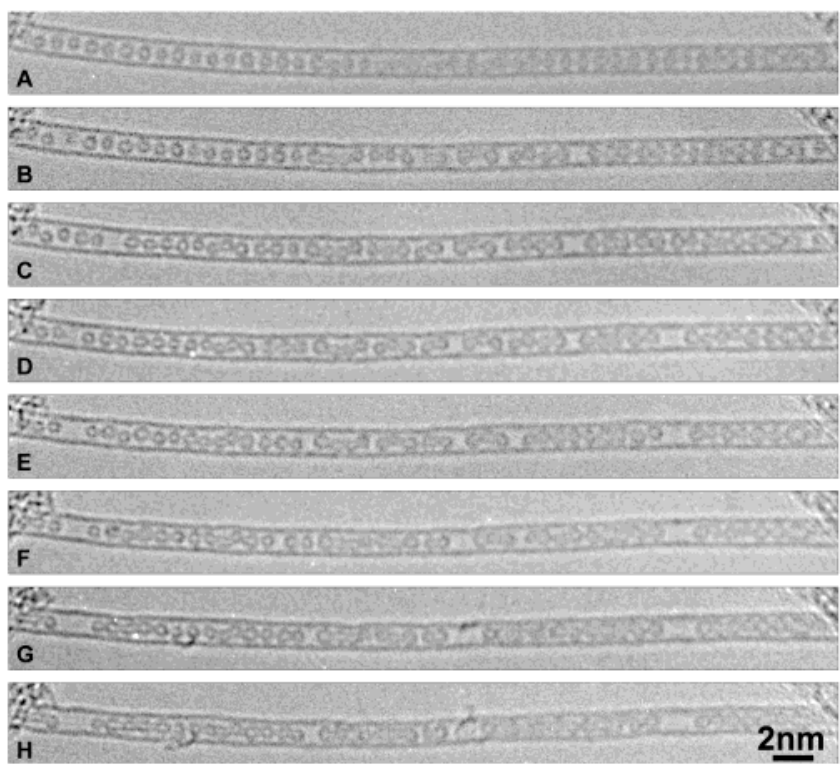

Figure 1. Sequence of TEM micrographs showing irradiationinduced coalescence of $\mathrm{C}_{60}$ within the lumen of an isolated SWNT. (a) Starting configuration after a minimal electron dose. $(b-h)$ Consecutive images recorded at 60-90 s intervals. Damage to the fullerenes occurs by the knock-on ejection of carbon atoms, resulting in a structural reconfiguration into corrugated tubules that are ca. 5-7 $\AA$ in diameter. These tubules exhibit regions of negative curvature that may be associated with the formation of heptagonal rings acting to link the discrete molecules together. Pronounced damage to the SWNT is also visible beginning in c, but its damage rate is appreciably slower because of curvature effects. Therefore, $\mathrm{C}_{60}-\mathrm{C}_{60}$ fusion predominates over $\mathrm{C}_{60}-\mathrm{SWNT}$ fusion.

belonging to the surrounding SWNT because of differences in strain energy. Because knock-on is a statistical event, it is not necessarily the case that every fullerene in a peapod will be damaged for very low electron doses (e.g., $\ll 10^{22} \mathrm{e}$ $\mathrm{cm}^{-2}$ ). Nevertheless, for reasonable doses, it becomes increasingly likely that all fullerenes will have suffered some atomic displacement. Therefore, irradiation under these conditions initially results in the formation of vacancies and/ or dangling bonds predominantly on the interior fullerenes. The damaged fullerenes can then merge with each other (and not with the substantially intact SWNT), reconstructing into more energetically stable tubular structures. This hypothesis was first asserted by two of us in an early peapod manuscript ${ }^{4}$ and is fully elucidated here.

We have undertaken both $\mathrm{MC}$ and MD simulations on a set of $\mathrm{C}_{60}$ molecules inside a $(10,10)$ nanotube. Because the large numbers of atoms in such systems preclude a direct ab initio treatment, we have opted for MD approaches based on a tight-binding Hamiltonian ${ }^{19,20}$ as well as empirical potentials. ${ }^{21}$ Both methods have proven to be remarkably accurate in most carbon-based systems. ${ }^{17,22-24}$ Our simulations were carried out at a series of temperatures, ranging from 1300 to $3600 \mathrm{~K}$. The simulations at high-temperature emulate the annealing-induced coalescence, albeit at temperatures higher than the experimental ones. These high temperatures are required to accelerate a process, which occurs over realistic time scales, the latter being beyond current computing capabilities. 

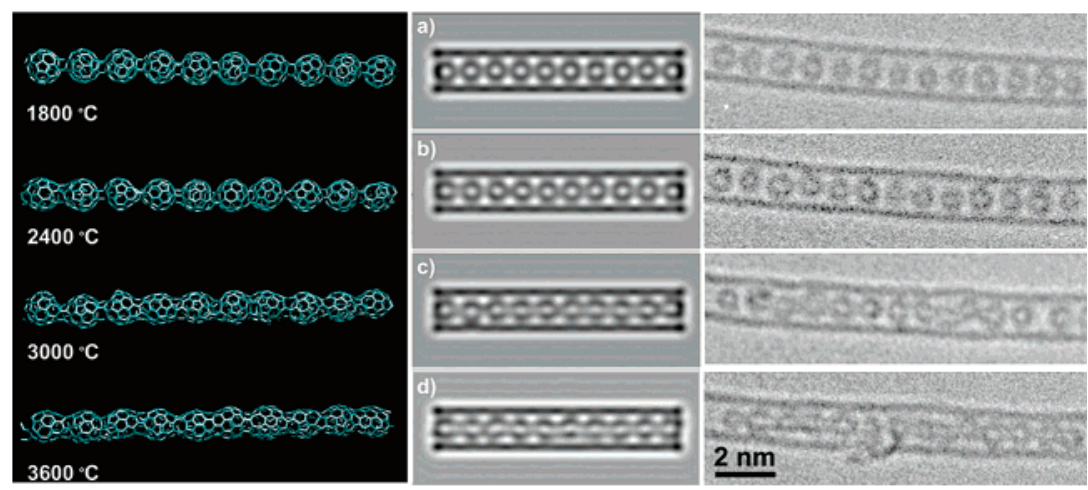

Figure 2. Left-panel images include MD simulations using the Tersoff potential of 10 complete $\mathrm{C}_{60}$ molecules inside an SWNT at (a) 1800 , (b) 2400, (c) 3000, and (d) $3600 \mathrm{~K}$ (note that the outer $(10,10)$ tube is not depicted for visualization purposes). The middle panel depicts the HRTEM simulations of the fullerene peapods at different temperatures (using a multislice method calculated with a Scherzer defocus). The right panel is the experimentally observed sequence under irradiation, in which the carbon cages polymerize, rearrange, and coalesce into a corrugated tubule.

The $\mathrm{C}_{60}$ molecules were randomly oriented and positioned inside the $(10,10)$ nanotube in such a way that their center of mass lay along the tube axis. In the case of the MD-Tersoff potential simulations, the effect of the encapsulating nanotube was included via an external confining potential of cylindrical symmetry. ${ }^{25}$ Within the present framework, a system consisting of 10 complete $\mathrm{C}_{60}$ molecules (no vacancies created) inside the nanotube was considered (Figure 2). The obtained configuration was simulated using canonical (constant volume, constant number of particles and constant temperature) MD using the Nosé-Poincaré algorithm ${ }^{26}$ for a total time of $300 \mathrm{ps}$ using a time step of $1 \mathrm{fs}$ at a temperature of 1600 $\mathrm{K}$. The last configuration obtained from this trajectory was used as a starting point for a subsequent simulation at 2400 $\mathrm{K}$. We repeated this process for a number of temperatures up to $3600 \mathrm{~K}$ by increasing the temperature in steps of 600 $\mathrm{K}$. Figure $2 \mathrm{a}$ illustrates the configuration obtained after simulation at $1800 \mathrm{~K}$ and those obtained from the dynamics at 2400,3000 , and $3600 \mathrm{~K}$. At $2400 \mathrm{~K}$, after $300 \mathrm{ps,} \mathrm{there}$ is no sign of coalescence (Figure 2b); rather, the fullerenes tend to polymerize, forming several $\mathrm{sp}^{3}$ bonds between each cage. As the temperature is increased, a remarkable tendency toward fullerene coalescence appears. At $3000 \mathrm{~K}$, regions of negative curvature containing heptagons as well as pentagons appear (Figure 2c), forming bottleneck structures between the fullerene cages, although the cages can still be resolved. As the temperature is further increased to $3600 \mathrm{~K}$, the resulting structure clearly evolves toward a corrugated tubular form (Figure 2d), which is reminiscent of a Haeckelite structure. ${ }^{14}$

We also performed simulated annealing calculations using the MC method ${ }^{17}$ in conjunction with the Metropolis algorithm ${ }^{27}$ and the many-body Tersoff potential, which has also provided accurate values of cohesive energies and elastic properties for carbon nanotubes. ${ }^{17}$ In this case, a system consisting of three complete $\mathrm{C}_{60}$ molecules (no atoms were extracted from the cages) located in a linear configuration was confined in a volume resembling a $(10,10)$ nanotube (Figure 3). At temperatures between 1000 and $4600 \mathrm{~K}$, we observed the polymerization of $\mathrm{C}_{60}$ units followed by their coalescence exhibiting heptagonal and octagonal carbon rings in a peanutlike configuration, which again consists of a corrugated tubular structure (Figure 3). To view the complete simulated $\mathrm{C}_{60}$ coalescence process, see the animation at http:// materials.ipicyt.edu.mx/mt_c60_qwer1234/.

To confirm the results obtained using a classical scheme, we have carried out tight-binding molecular dynamics simulations (TBMD) in a super cell consisting of four $\mathrm{C}_{60}$ molecules encapsulated in a $(10,10)$ tube at $1500 \mathrm{~K}$ (Figure 4). In this calculation, the atoms of both the fullerene and nanotube were explicitly considered (880 atoms). Eight carbon atoms (i.e., two per molecule) were extracted randomly from the cages, thus creating vacancies that are produced under electron irradiation by knock-on effects. After a simulation time of about 2 ps, the carbon molecules polymerize, and after $50 \mathrm{ps}$, they clearly coalesce, forming corrugated tubules exhibiting heptagonal, octagonal, and hexagonal rings similar to those obtained in the previous calculations (Figure 4).

The latter results confirm the tendency of fullerene molecules (with or without vacancies) to coalesce within confined environments (e.g., nanotubes) at specific temperatures. HRTEM image simulations on all of these models of corrugated tubular structures reveal remarkable similarities with those observed experimentally (Figure 5) in fullerenes merged inside peapods under either electron irradiation (vacancies induced) (Figure 1) or thermal annealing (no vacancies likely to be induced). In addition, all of the present calculations lead to final configurations consisting of corrugated (nongraphitizable) carbon tubules.

To verify the stability of these slightly corrugated tubes generated by the coalescence of $\mathrm{C}_{60}$ in peapods, we have investigated the energetics of some peculiarly symmetric coalescence geometries that lead to interesting topologies. Figure 6 illustrates three corrugated tubes created by the coalescence of $\mathrm{C}_{60}$ along the (a) 2-fold axis (T2), (b) 3-fold axis (T3), and (c) 5-fold axis (T5). T2 exhibits pentagonal, hexagonal, and heptagonal rings of carbon, and T3 and T5 possess pentagons, hexagons, and octagons. The structural optimization of these three structures was performed using ab initio calculations. ${ }^{28}$ The T3 structure (Figure $6 \mathrm{~b}$ inset) is found to be the most stable, with a cohesive energy of 374 


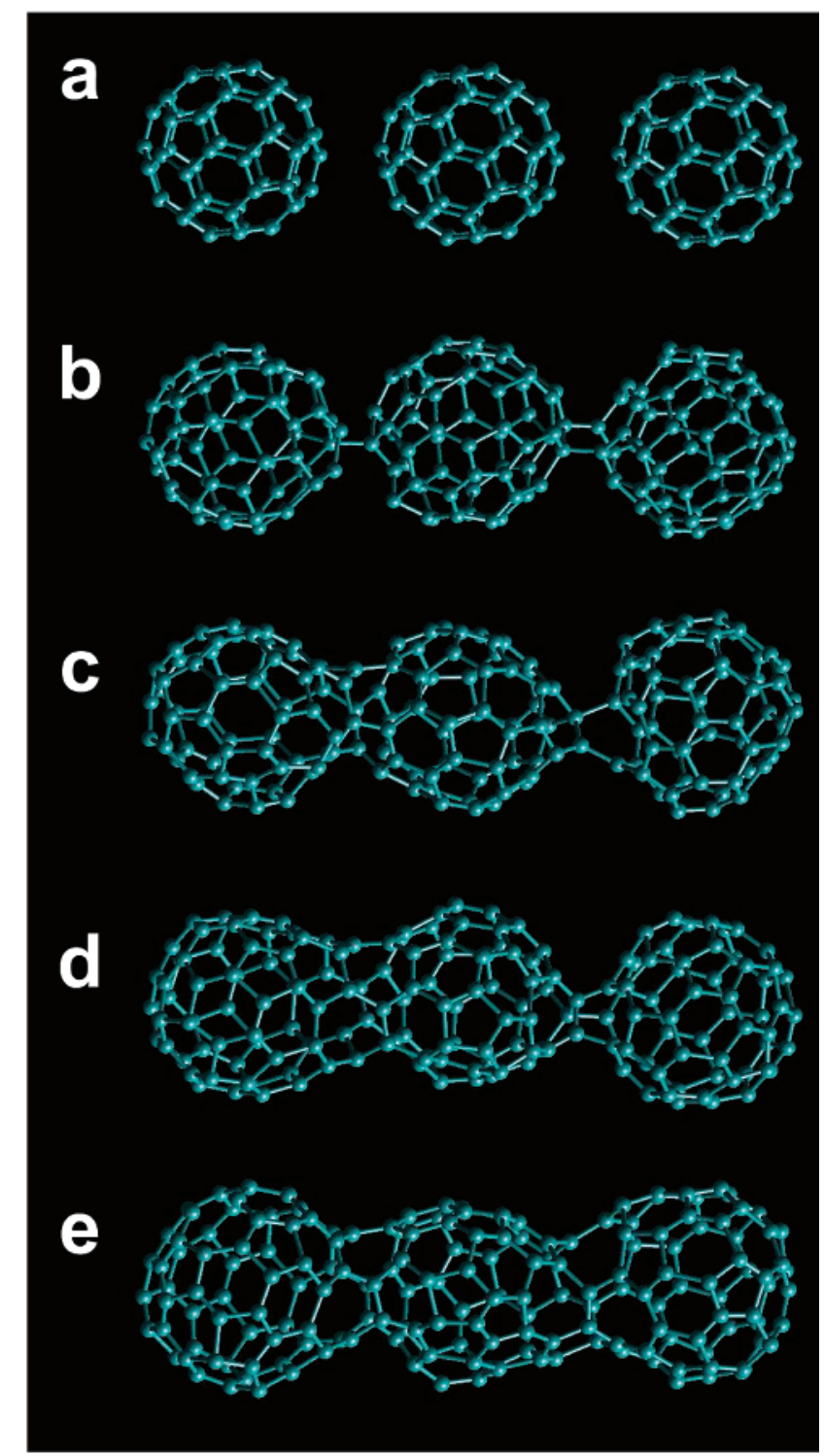

Figure 3. $(a-d)$ Molecular models of three aligned $\mathrm{C}_{60}$ fullerenes (no vacancies created) obtained using the MC method in conjunction with the Metropolis algorithm and the many-body Tersoff potential. The results indicate that at $4600 \mathrm{~K}$ the cages merge and form a corrugated tube exhibiting heptagonal and octagonal rings linking the structures. (See http://materials.ipicyt. edu.mx/mt_c60_qwer1234/.)

meV/atom higher than that of a single planar graphite sheet. The cohesive energies of the T5 structure (Figure $6 \mathrm{c}$ inset) and the T2 structure (Figure 6a inset) are $391 \mathrm{meV} / \mathrm{atom}$ and $580 \mathrm{meV} / \mathrm{atom}$, respectively, as compared to that of graphene.

To predict the electronic properties of these corrugated tubes, ab initio band structures were also calculated for the three molecular models. The T3 structure is found to display semiconducting behavior with a direct band gap of $1.65 \mathrm{eV}$ at the $\Gamma$ point ( $1.1 \mathrm{eV}$ in the tight-binding approximation). The other two forms (T5 and T2) are found to be metallic with four and two bands crossing the Fermi energy, respectively. The interesting diversity in the electronic band structures of these corrugated tubes naturally led us to

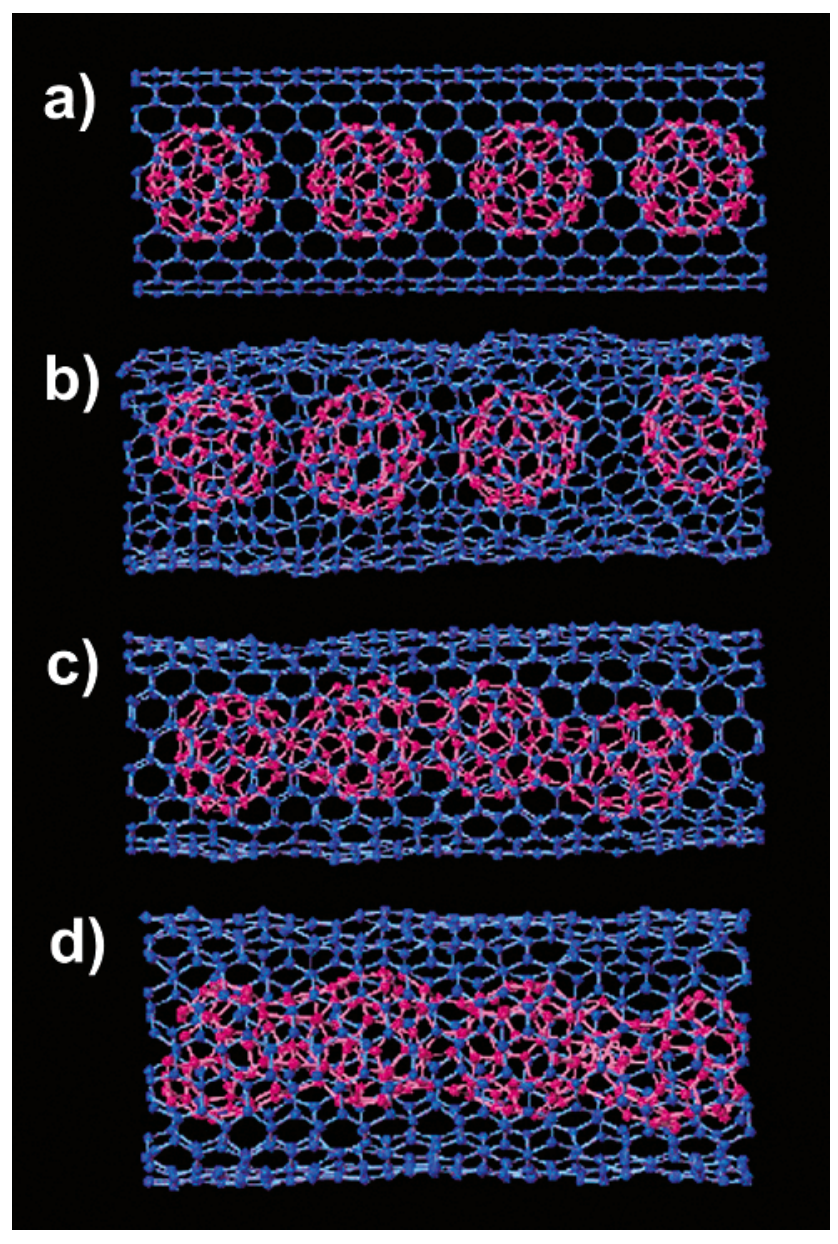

Figure 4. Sequence of TBMD simulations of fullerene coalescence inside a $(10,10)$ nanotube (diameter ca. $1.38 \mathrm{~nm}$ ) at $1500 \mathrm{~K}$. TBMD calculations were performed using a time step of $0.7 \mathrm{fs}$ to ensure an optimal integration of equations of motion and energy conservation with a total simulation time of 80 ps. (a) Simulation starts with four $\mathrm{C}_{60}$ molecules placed inside an SWNT. The unit cell contains 640 carbon atoms (blue spheres) for the nanotube and 240 carbon atoms for the encapsulated fullerenes. Periodic boundary conditions are imposed along the tube axis. (b) Two carbon atoms were extracted from each $\mathrm{C}_{60}$ molecule to promote the merger. The system is also slow at $1500 \mathrm{~K}$. After less than a couple of picoseconds, the cages tend to get closer to each other. (c) After $20 \mathrm{ps}$, links are clearly established among the cages, thus resulting in a defective tube. (d) After $50 \mathrm{ps}$, the cages have reconstructed into a corrugated tubular structure. To get an infinite periodic system, the length of the surrounding tubular shell has been reduced to 520 carbon atoms (13 unit cells of a $(10,10)$ tube). The reconstructed surface, after connection between the two ends, is highly defective and contains a large number of topological defects.

investigate the electronic transport properties of these novel forms of carbon.

Quantum conductance in corrugated tubules has been evaluated using Green's function and a transfer-matrix-based approach for computing electron transport in these extended systems. ${ }^{29}$ A Slater-Koster tight-binding $\mathrm{sp}^{3}$ Hamiltonian for carbon was used in the present study. The results are illustrated in Figure 6. The general trend observed in the ab intio band-structure calculations is fully confirmed. The T3 structure (Figure $6 \mathrm{~b}$ ) is found to be zero, as expected. However, $4 G_{0}$ (Figure 6c) and $2 G_{0}$ (Figure 6a) values $\left(G_{0}\right.$ 

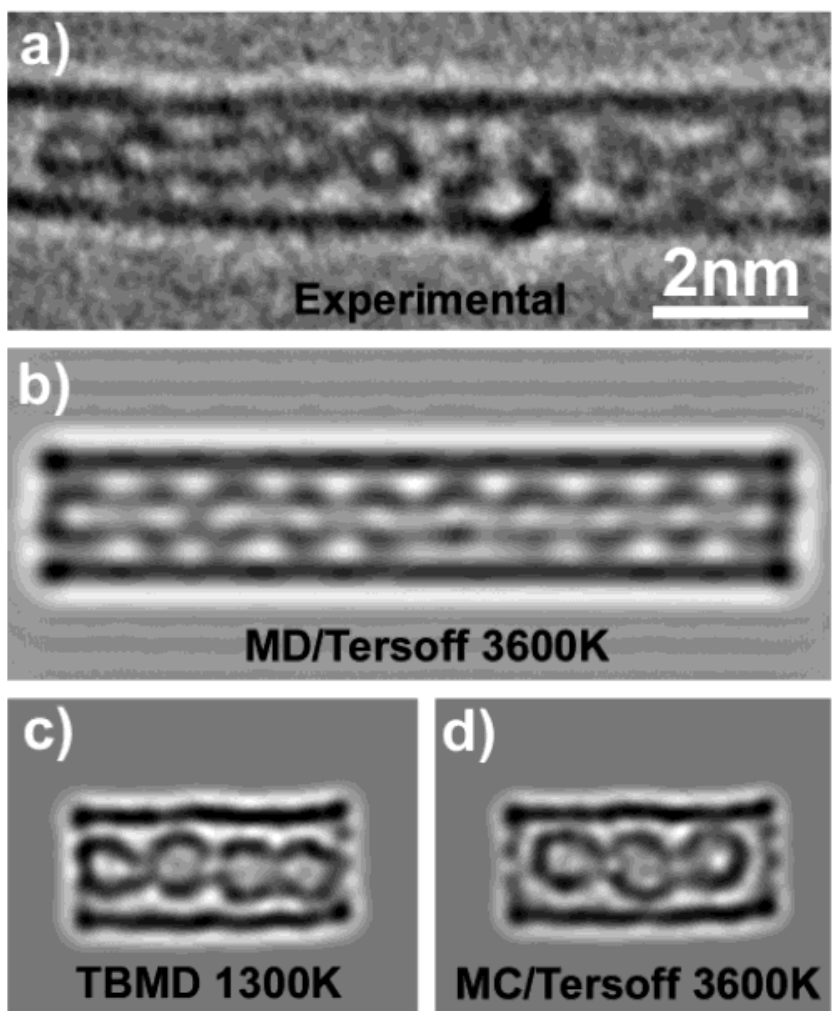

Figure 5. (a) HRTEM image of coalesced fullerenes inside an SWNT observed during electron irradiation in TEM. $(b-d)$ Molecular simulations of the final configurations obtained using three different theoretical approaches. (b) MD/Tersoff potential at $3600 \mathrm{~K}$. (c) TBMD calculations at $1300 \mathrm{~K}$. (d) MC-thermal annealing/Tersoff potential obtained at $3600 \mathrm{~K}$. In all cases, we observed that the simulations resemble the results obtained experimentally under electron irradiation (a). Simulated HRTEM images were calculated using a multislice method with a Scherzer defocus.

$\left.=2 e^{2} / h\right)$ are calculated for the $\mathrm{T} 5$ and $\mathrm{T} 2$ structures, illustrating the electron-transport diversity in these corrugated systems. Consequently, investigating the electronic properties of the coalesced fullerene peapods is an important tool in extracting information on the topology of carbon systems.

Our theoretical investigations and experimental observations suggest that the coalescence of $\mathrm{C}_{60}$ molecules within SWNTs is obtained along two main avenues.

By Electron Irradiation. In this case, carbon atoms are displaced from the cages by knock-on effects. This results in the creation of dangling bonds and vacancies, which in turn can anneal to minimize the total energy of the system. The annealing process results in the coalescence of the fullerene cages and, finally, in the formation of a small-diameter corrugated nanotube. This nanotube is characterized by the presence of a number of heptagonal or octagonal rings acting as links between the original fullerene molecules. It is clear that the cagelike molecules do not necessarily need to have the same orientation with respect to the tube axis. Note that it would be difficult to anneal the heptagons and octagons with pentagonal rings to achieve a perfect hexagonal tubular network. Therefore, these new types of carbon tubules can be considered to be nongraphitizable nanocarbons.

By Thermal Annealing. For fullerene molecules that are randomly oriented inside an SWNT, fullerenes can merge

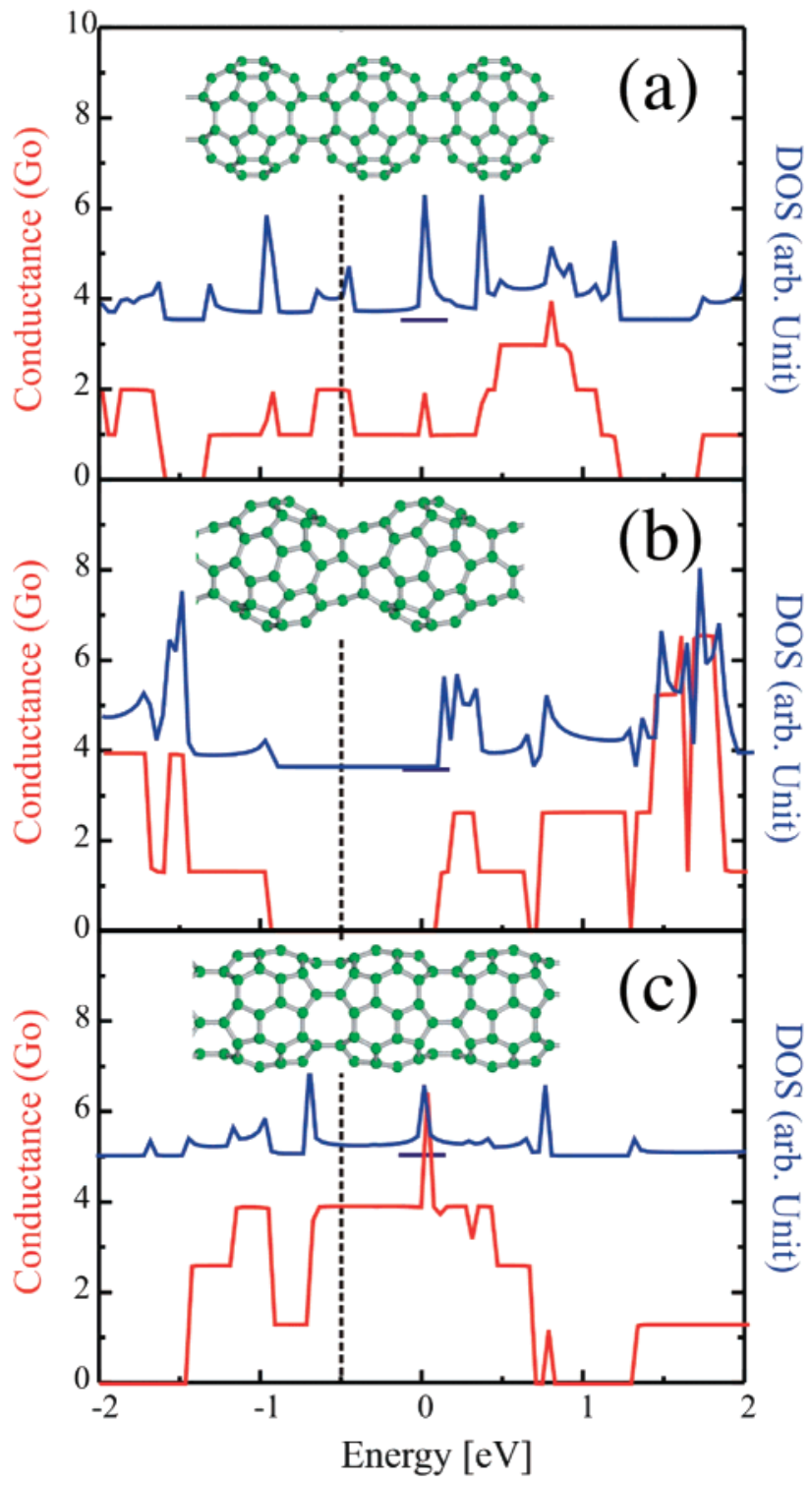

Figure 6. $\sigma+\pi$ conductance (red) and TB-DOS (blue) spectra of three coalesced fullerene peapods. The coalesced systems display (a) a 2-fold axis, (b) a 3-fold axis, and (c) a 5-fold axis along the outer nanotube main axis, as shown in the insets. The electronic transport properties depend strongly on the topology of the coalesced fullerene tubes, and metallic $(\mathrm{a}, \mathrm{c})$ and semiconducting (b) characteristics are found. The small horizontal bars indicate the zero-density-of-states levels. The Fermi levels are indicated by vertical dotted lines. The DOS and conductance are respectively expressed in arbitrary units (right) and $G_{0}$ units (left), where $G_{0}=$ $2 e^{2} / h$ is the quantum of conductance ( $e$ is electronic charge and $h$ is Planck's constant).

without removing any atom by heat treatment, provided enough thermal energy is applied. The system can then reach a more stable configuration through a process driven by the minimization of the surface energy. This stable state will mainly consist of coalesced cages joined by heptagonal, octagonal, or larger carbon rings characterized by local negative curvature.

It is important to note that the formation of these corrugated tubes, which resemble the theoretically proposed Haeckelite structures, could also explain the observation of critical superconducting temperatures higher than those in 
conventional SWNTs. ${ }^{13,30,31}$ The possibility of producing small-diameter superconducting nanotubes containing a high density of nonhexagonal rings will be the subject of further study.

In conclusion, through a combination of experimental observations and theoretical calculations, we have demonstrated that fullerene molecules can easily coalesce inside SWNTs via a process driven by either thermal annealing or electron irradiation. The resulting structure consists of a corrugated tubule nested inside the original SWNT. This novel carbon nanostructure exhibits pentagonal, hexagonal, heptagonal, and octagonal rings and resembles the theoretically proposed Haeckelite structure. Using first principles calculations on models of crystalline corrugated tubules (formed after $\mathrm{C}_{60}$ coalescence), we have predicted stability and different electronic behavior depending on the topology. We envision that novel nanostructured carbon systems can be produced following different sequences of fullerene coalescence encapsulated in porous materials with different morphologies, thus opening the way to new candidates for nanoelectronics.

Acknowledgment. We are indebted to Daniel Ramírez and E. Floresgomez for technical assistance. We also thank the Spanish Ministry of Science and Technology (project BFM2002-03278(E.H.)), CONACYT-México (grants W-8001millennium initiative (H.T., M.T.), G-25851-E (H.T., M.T.), 36365-E (H.T.), 37589-U (M.T.)); the Belgian interuniversity project (PAI5/1/1) on Quantum Size Effects in NanoStructured Materials (J.C.C.), the National Fund for Scientific Research (FNRS) of Belgium (V.M., J.C.C.), the Communauté Française de Belgique through the Action de Recherche Concertée entitled Interaction Électron-Vibration dans les Nanostructures (J.C.C.), the Mathematical Information and Computational Sciences Division, Office of Advanced Scientific Computing Research of the U.S. Department of Energy (contract no. DE-AC05-00OR22725) with UT-Battelle, LLC (V.M.), the Alexander von Humboldt Stiftung (M.T.), and the European Union: CNT-NET project (contract no. G5RT-CT2001-05026 (M.T.)) for financial support. Work at the University of Pennsylvania was supported through a DURINT grant from the Office of Naval Research (no. N00014-01-1-0657). Work at North Carolina State University was supported in part by the Petroleum Research Fund of the American Chemical Society.

\section{References}

(1) Kroto, H. W.; Heath, J. R.; O’Brien, S. C.; Curl, R. F.; Smalley, R. E. Nature 1985, 318, 162-163.

(2) Iijima, S. Nature 1991, 354, 56-58.

(3) Smith, B. W.; Monthioux, M.; Luzzi, D. E. Nature 1998, 396, 323324.

(4) Smith, B. W.; Luzzi, D. E. Chem. Phys. Lett. 2000, 321, 169-174.

(5) Burteaux, B.; Claye, A.; Smith, B. W.; Monthioux, M.; Luzzi, D. E.; Fischer, J. E. Chem. Phys. Lett. 1999, 310, 21-24.

(6) Sloan, J.; Dunin-Borkowski, R. E.; Hutchison, J. L.; Coleman, K S.; Clifford Williams, V.; Claridge, J. B.; York, A. P. E.; Xu, C.; Bailey, S. R.; Brown, G.; Friedrichs, S.; Green, M. L. H. Chem. Phys. Lett. 2000, 316, 191-198.
(7) Zhang, Y.; Iijima, S. Philos. Mag. Lett. 2000, 80, 427-433.

(8) Suenaga, K.; Tence, T.; Mory, C.; Colliex, C.; Kato, H.; Okazaki, T.; Shinohara, H.; Hirahara, K.; Bandow, S.; Iijima, S. Science 2000, 290, 2280-2283.

(9) Hirahara, K.; Suenaga, K.; Bandow, S.; Kato, H.; Okazaki, T.; Shinohara, H.; Iijima, S. Phys. Rev. Lett. 2000, 85, 5384-5387.

(10) Lee, J.; Kim, H.; Kahng, S. J.; Kim, G.; Son, Y. W.; Ihm, J.; Kato, H.; Wang, Z. W.; Okazaki, T.; Shinohara, H.; Kuk, Y. Nature 2002, $415,1005-1008$.

(11) Smith, B. W.; Luzzi, D. E.; Achiba, Y. Chem. Phys. Lett. 2000, 331, 137-142.

(12) Yeretzian, C.; Hansen, K.; Diederich, F.; \& Whetten, R. L. Nature 1992, 359, 44-47.

(13) Tang, Z. K.; Zhang, L. Y.; Wang, N.; Zhang, X. X.; Wen, G. H.; Li, G. D.; Wang, J. N.; Chan, C. T.; Sheng, P. Science 2001, 292, 24622465.

(14) Terrones, H.; Terrones, M.; Hernández, E.; Grobert, N.; Charlier, J. C.; Ajayan, P. M. Phys. Rev. Lett. 2000, 84, 1716-1719.

(15) Berber, S.; Kwon, Y. K.; Tománek, D. Phys. Rev. Lett. 2002, 88 , $185502-185505$.

(16) Zhao, Y. F.; Yakobson, B. I.; Smalley, R. E. Phys. Rev. Lett. 2002, 88, 185501-185504. Zhao, Y. F.; Smalley, R. E.; Yakobson, B. I. Phys. Rev. B 2003, 66, 195409.

(17) Terrones, M.; Terrones, H.; Charlier, J. C.; Banhart, F.; Ajayan, P. M. Science 2000, 288, 1226-1229.

(18) Smith, B. W.; Luzzi, D. E. J. Appl. Phys. 2001, 90, 3509-3515.

(19) Xu, C. H.; Wan, C. Z.; Chan, C. T.; Ho, K. M. J. Phys.: Condens. Matter 1992, 4, 6047-6054.

(20) Goedecker, S.; Colombo, L. Phys. Rev. Lett. 1994, 73, 122-125.

(21) Tersoff, J. Phys. Rev. Lett. 1988, 61, 2879-2882.

(22) Maiti, A.; Brabec, C. J.; Roland, C.; Bernholc, J. Phys. Rev. B 1995, $52,14850-14858$.

(23) Terrones, H. Fullerene Sci. Technol. 1995, 3, 107-114.

(24) Buongiorno Nardelli, M.; Yakobson, B. I.; Bernholc, J. Phys. Rev. B 1998, 57, R4277-R4280.

(25) The confining potential that was used was obtained by assuming that the encapsulating nanotube consisted of a uniform distribution of carbon atoms. The interaction with the confined fullerene atoms was assumed to be of a Lennard-Jones type, with parameters given by Grifalco and Lad (J. Chem. Phys. 1956, 25, 693). The confining potential resulted from integrating the contribution from each surface element over the surface area of the nanotube. The resulting potential depends only on the positions of the fullerene atoms with respect to the axis of the confining nanotube.

(26) Bond, S. D.; Leimkuhler, B. J.; Laird, B. B. J. Comput. Phys. 1999, 151, 114-134.

(27) Metropolis, N.; Rosenbluth, A.; Rosenbluth, M.; Teller, A.; Teller, E. J. Chem. Phys. 1953, 21, 1087-1092.

(28) In this work, ab initio calculations were performed within the formalism of density functional theory (DFT) in the local density approximation (LDA). The interaction between valence electrons and ionic cores was described using norm-conserving pseudopotentials for carbon (Troullier, N.; Martins, J. L. Phys. Rev. B 1991, 43, 1993). Periodic boundary conditions were adopted along the axis of the tube and keeping a minimum $4.5-\AA$ distance between repeated images along the two other directions. The electronic wave functions were expanded into plane waves with kinetic energy cutoffs of $50 \mathrm{Ry}$. Three $\mathrm{k}$ points were used to sample the 1D Brillouin zone.

(29) Buongiorno Nardelli, M.; Bernholc, J. Phys. Rev. B 1999, 60, R16338-R16341.

(30) Kociak, M.; Kasumov, A. Y.; Gueron, S.; Reulet, B.; Khodos, II.; Gorbatov, Y. B.; Volkov, V. T.; Vaccarini, L.; Bouchiat, H. Phys. Rev. Lett. 2001, 86, 2416-2419.

(31) Kasumov, A. Y.; Deblock, R.; Kociak, M.; Reulet, B.; Bouchiat, H.; Khodos, II.; Gorbatov, Y. B.; Volkov, V. T.; Journet, C.; Burghard, M. Science 1999, 284, 1508-1511.

NL034283F 